\section{Spectral characteristics of the internal jugular vein and central venous pressure pulses: a proof of concept study}

\author{
Clive Beggs, ${ }^{1}$ Valentina Tavoni, ${ }^{2}$ \\ Erica Menegatti, ${ }^{2,3}$ Mirko Tessari, ${ }^{2}$ \\ Luca Giovanardi, ${ }^{4}$ Riccardo Ragazzi, ${ }^{3}$ \\ Anna Maria Malagoni ${ }^{2}$ \\ ${ }^{1}$ Institute for Sport, Physical Activity \\ and Leisure, Leeds Beckett University, \\ Leeds, UK; ${ }^{2} \mathrm{Hub}$ Centre for Venous and \\ Lymphatics Diseases of Emilia Romagna \\ Region, University Hospital of Ferrara, \\ Ferrara, Italy; ${ }^{3}$ Department of \\ Translational Medicine and for \\ Romagna, University of Ferrara, \\ Ferrara, Italy; ${ }^{4}$ Service of Clinical \\ Engineering, University Hospital of \\ Ferrara, Ferrara, Italy
}

\begin{abstract}
In this proof-of-concept study the impact of central venous pressure (CVP) on internal jugular veins cross-sectional area (CSA) and blood flow time-average velocity (TAV) was evaluated in eight subjects, with the aim of understanding the drivers of the jugular venous pulse. CVP was measured using a central venous catheter while CSA variation and TAV along a cardiac cycle were acquired using ultrasound. Analysis of CVP, CSA and TAV time-series signals revealed TAV and CSA to lag behind CVP by on average 0.129 $\mathrm{s}$ and $0.138 \mathrm{~s}$, with an inverse correlation between CSA and TAV $(r=-0.316)$. The respective autocorrelation signals were strongly correlated (mean $\mathrm{r}=0.729-0.764$ ), with mean CSA periodicity being $1.062 \mathrm{~Hz}$. Fourier analysis revealed the frequency spectrums of CVP, TAV and CSA signals to be dominated by frequencies at approximately 1 and $2 \mathrm{~Hz}$, with those $>1 \mathrm{~Hz}$ greatly attenuated in the CSA signal. Because the autocorrelograms and periodograms of the respective signals were aligned and dominated by the same underlying frequencies, this suggested that they are more easily interpreted in the frequency domain rather than the time domain.
\end{abstract}

\section{Introduction}

Evaluation of the jugular venous pulse (JVP), defined as the movement or expansion of the jugular veins due to changes in pressure in the right atrium, provides valuable information about cardiac hemodynamics and filling pressures, ${ }^{1}$ as well as yielding characteristic wave patterns indicative of cardiac disease. ${ }^{2}$ As such, the JVP can be used to provide an indirect estimate of the central venous pressure (CVP) ${ }^{3,4}$ Moreover, it is also recognized that JVP responds to changes in cerebrospinal fluid flow and intracranial blood flow over the cardiac cycle (CC), making it a parameter of pivotal importance when evaluating cerebrovascular circulation. ${ }^{5-7}$ JVP is traditionally evaluated through visual inspection of changes in volume of the internal jugular vein (IJV) which can also provide an estimate of the $\mathrm{CVP},{ }^{3}$ albeit with a low accuracy. ${ }^{8,9}$ The most reliable and broadly used method to assess the CVP is the direct catheterization of the IJV or subclavian veins. ${ }^{10}$ However, this is an invasive maneuver with an associated risk of complications and thus is not considered feasible as a routine approach. ${ }^{11}$ Recently, it has been shown that the JVP can be obtained using B-mode ultrasound (US) assessment of changes in the cross-sectional area (CSA) of the IJVs over the CC. ${ }^{12,13}$ Significant correlations have also been reported between CVP and IJV-CSA, ${ }^{14-}$ ${ }^{16}$ suggesting that US assessment of the JVP might have potential as a tool for non-invasively determining CVP. However, the information contained in the JVP is difficult to interpret, with relatively little known about the effect that changes in CVP and IJV-CSA have on blood flow in the IJVs. ${ }^{17}$ Consequently, much remains unknown about the dynamics of the JVP and its interaction with CVP. With this in mind, we established a program of work to investigate the potential for using US assessment of the JVP to noninvasively evaluate CVP. ${ }^{18}$ As part of this program we undertook the proof-of-concept study presented here, which utilized a novel autocorrelation function, with the specific aim of understanding how changes in CVP over the CC influence blood flow and vessel CSA in the IJVs. In particular, we wanted to better understand the cardiac drivers of the JVP. To this end we performed spectral analysis of blood flow, vessel volume and CVP data acquired from a sample of hospital patients.

\section{Materials and Methods}

This study is part of a project granted by the Italian Ministry of Health (Ricerca Finalizzata 2013, \#RF-2013-02358029) that was approved by the Ethics Committee of Ferrara, Italy (reference No.160499) and was carried out in accordance with the ethical guidelines on good clinical practice as laid down in European Directive and the
Correspondence: Anna Maria Malagoni, Vascular Diseases Center, University Hospital of Ferrara, Via Aldo Moro 8, 44124 Ferrara, Italy.

Tel.: +39.0532.239783 - Fax: +39.0532.237582. E-mail:mlgnmr@unife.it

Key words: Jugular venous pulse; central venous pressure; spectral signals; ultrasound.

Funding: this study was supported by the Italian Ministry of Health Grant: Ricerca Finalizzata 2013, \#RF-2013-02358029.

Contributions: $\mathrm{CB}$ conception and design; analysis and interpretation of data; drafting the manuscript; revising the manuscript critically for important intellectual content; final approval of the manuscript submitted; VT, LG, RR, data collection; analysis and interpretation of data; revising the manuscript critically for important intellectual content; final approval of the manuscript submitted; EM, MT, data collection; revising the manuscript critically for important intellectual content; final approval of the manuscript submitted; AMM, conception and design; data collection; analysis and interpretation of data; drafting of the manuscript; revising the manuscript critically for important intellectual content; final approval of the manuscript submitted.

Conflict of interests: the authors declare no potential conflict of interests.

Conference presentation: data were partially presented at $9^{\text {th }}$ Annual Meeting of the International Society of Neurovascular Disease, May $30^{\text {th }}-31^{\text {st }}, 2019$, Ferrara, Italy.

Availability of data and materials: all data generated or analyzed during this study are included in this published article.

Ethical approval and consent to participate: this study was approved by the Ethics Committee of Ferrara, Italy (reference No.160499) and was carried out in accordance with the ethical guidelines on good clinical practice as laid down in European Directive and the Declaration of Helsinki. All participants signed a written informed consent form.

Received for publication: 7 March 2021 Accepted for publication: 9 March 2021

This work is licensed under a Creative Commons Attribution 4.0 License (by-nc 4.0).

COCopyright: the Author(s), 2021

Licensee PAGEPress, Italy

Veins and Lymphatics 2021; 10:9732

doi:10.4081/vl.2021.9732 
Declaration of Helsinki.

\section{Subjects}

Ten consecutive hospitalized adult patients ( 2 males, 8 females, age $63 \pm 10$ years) at University Hospital of Ferrara requiring a scheduled central venous catheterization and measurement of the CVP as part of their usual care were enrolled for this study. Inclusion criteria were: age $>18$ years, spontaneous breathing and capacity to give informed consent. Patients were excluded if they needed a cannulation via the internal jugular venous access in order to not interfere with the ultrasound assessment. All participants signed a written informed consent form.

\section{Central venous pressure measurement}

In a surgery room, patients undertook a subclavian tunneled catheterization. Following the catheterization, patients were placed out of the surgery room lying completely supine on a bed in order to measure the CVP according to the standard. ${ }^{19}$ In addition, three electrodes were placed on patients' chests for the simultaneous assessment of the ECG signal. The pressure line and the ECG cables were connected to a standard analogic monitor (Philips M3046A M4, Philips Medical System, Boeblingen, Germany) which in turn was connected to a video grabber system, allowing the capture and storage of the screen images on a computer, as they were not directly accessible in their digital format. All measurements were performed by a single investigator.

\section{Ultrasound assessment of the inter- nal jugular veins}

Alongside the direct CVP measurement, the US assessment of the IJVs was performed as previously described ${ }^{12,13,18}$ by means of a Vivid-q ultrasound system (GE Medical Systems, Horten, Norway) equipped with a linear array probe L12-RS (7.5-11 MHz). Before that assessment three electrodes were placed on patients' chests and connected to the ultrasound system for simultaneously measuring the ECG signal. Patients had to maintain their neck position held on a longitudinal axis, while the operator placed the probe in a transverse plane with respect to the length of the vessels, at the level of C5-C6, in order to insonate the IJV-CSA. Moreover, time average velocity (TAV) was evaluated at the same venous location through the measurement of the Doppler PW trace assessed in the longitudinal scan. ${ }^{12,13,17}$ A B-mode video-clip of 10$15 \mathrm{sec}$, including the ECG trace, was recorded and stored. All the US evaluations were performed by a single investigator.

\section{Post-processing}

The stored US-IJV video clips and the CVP images were processed off-line to obtain numerical dataset to enable the subsequent analyses and formulation of models as previously described. ${ }^{12,13,17,18,20}$ Firstly, images and video clips were transformed using the software ImageJ (ImageJ, U.S. National Institutes of Health, Bethesda, MD, USA), which allows the measurement of, frame per frame, area (pixel), perimeter (pixel) and grey level of a selected region of interest. The procedure to obtain an IJVCSA dataset of signals over time consisted of many steps both manual and automatic. $12,13,17,18,20$ This procedure provided the IJV-CSA values in $\mathrm{cm}^{2}$ versus sonogram acquisition time, with the obtained result corresponded to the JVP trace. Similar steps were followed to analyze the CVP and TAV images as previously reported. ${ }^{20}$ The time series signal dataset was produced by digitally identifying the position of each point of the trace represented on the acquired images. Subsequently the JVP, the CVP and TAV traces were synchronized using the ECG signal recorded together with the measurements. The obtained datasets were then elaborated using MATLAB (The MathWorks, Inc. version 8.3.0.532 (R2014a), Natick, MA, USA) and R software ( $R$ core Team 2013, $R$ foundation for Statistical Computing, Vienna, Austria) to remove most of the frequencies of non-cardiac origin, mainly CSA variations due to activation of the thoracic pump. ${ }^{21}$ Finally, in order to allow easier comparison, the length of all the traces of each subject was conformed and brought into line with the others by dividing each trace in accordance to the cardiac cycle and extending them by the same number of frames.

\section{Data analysis}

Signal processing and statistical analysis were undertaken using in-house algorithms written in $\mathrm{R}$ software. The relationship between the CSA, TAV and CVP time series signals for the respective subjects were assessed using Pearson correlation analysis, with the time lags between the signals computed using the 'ccf' cross-correlation function in R. Differences between the signal time lags were evaluated using a paired t-test, with $\mathrm{P}<0.05$ deemed to be significant. For each subject, the autocorrelation signals were derived from the CSA, TAV and CVP time series signals using the 'acf' function in R, and the r-values between the respective autocorrelation signals computed. Autocorrelation, which assesses the degree of similarity between a given time series signal and a lagged version of itself over successive time intervals, can be used to gain insights into the spectral characteristics of signals. Through the Wiener-Khinchin theorem it can be shown that the Fourier transform (used in Fourier analysis) is closely related to the autocorrelation function of a signal. ${ }^{22}$ As such, the autocorrelation signal was used to compute the overall periodicity of the IJVCSA time series signal using Equation 1.

$f=1 / D \times d t$

Where $f$ is the periodicity (dominant frequency) of the time series signal; $D$ is the lag distance between successive peaks in the autocorrelogram; and $d t$ is the sampling interval. Finally, spectral analysis was performed on the respective time-series signals using the ' $\mathrm{fft}$ ' Fourier transform function in $\mathrm{R}$ to produce periodograms for each subject. From these the dominant frequencies (with amplitudes) were extracted for each subject.

Post-processing phase and data analysis were performed by two investigators blinded to the demographics and clinical conditions of the subjects.

\section{Results}

Video clips and images of eight subjects (age $=62 \pm 11$ years; 1 male $/ 7$ females) were processed and analyzed. The US video clips and CVP images of two subjects were excluded from the post-processing because they showed technical imperfections (CSA edges not perfectly outlined and CVP images not readable). For the purposes of this study only the right IJV US video clip per each subject was chosen and processed. The right IJV was chosen because it is closer to the heart than the left IJV and as such it is directly connected to the right atrium. The descriptive statistical results for the IJV-CSA, TAV and CVP time-series signals are presented in Table 1.

These reveal that the average mean, median and standard deviation (SD) values for the respective signals were: CVP (mean $=6.59 ; \quad$ median $=6.66 ; \quad \mathrm{SD}=1.08$ $\mathrm{mmHg}$ ); TAV (mean=10.97; median=11.32; $\mathrm{SD}=5.72 \mathrm{~cm} / \mathrm{s}$ ); and CSA (mean=0.99; median $\left.=0.99 ; \mathrm{SD}=0.06 \mathrm{~cm}^{2}\right)$. The results of the correlation analysis are presented in Table 2. From these correlations a complex picture emerges. While the relationship between the CSA and TAV signals was largely an inverse one (mean (SD) $\mathrm{r}=-0.316(0.396)$; all $\mathrm{P}<0.001$ ), with only two subjects exhibiting a positive correlation, the correlations between the CVP and TAV, and CVP and CSA were much more inconsistent. For example, for the CVP and CSA signals four subjects exhibited a significant negative correlation $(\mathrm{P}<0.001)$, 
while four had a positive correlation $(\mathrm{P}<=0.001$ for three of these). Likewise, the correlations between the CVP and TAV were equally mixed, with four positive and four negative (all $\mathrm{P}<0.001$ ). However, when the respective signals were aligned, then the correlations (i.e., the lag correlations) became much stronger [CVP and TAV: mean (SD) $\mathrm{r}=$ -0.514 (0.286); CVP and CSA: mean (SD) $\mathrm{r}=0.496(0.272)$; and CSA and TAV: mean (SD) $\mathrm{r}=-0.530(0.254)]$, with the TAV and CSA signals lagging behind the CVP signal by $0.129 \mathrm{~s}(\mathrm{SD}=0.066 \mathrm{~s})$ and $0.138 \mathrm{~s}$ $(\mathrm{SD}=0.071 \mathrm{~s})$ respectively. However, the dif- ference between these two-time lags was not significant $(\mathrm{P}=0.783)$. Although the correlation results for the CVP, TAV and CSA timeseries signals were mixed, those for their autocorrelation counterparts (Table 3) were generally highly positively correlated [CVP and TAV: mean (SD) r=0.764 (0.217); CVP

Table 1. Descriptive statistics for the and central venous pressure, time-average velocity and internal jugular vein cross-sectional area signals from the respective subjects.

\begin{tabular}{|c|c|c|c|c|c|c|c|c|c|c|c|c|c|c|c|}
\hline ID & $\begin{array}{c}\text { CVP } \\
\text { Mean } \\
\left(\mathrm{CmH}_{2} \mathrm{O}\right)\end{array}$ & $\begin{array}{c}\text { CVP } \\
\mathrm{SD} \\
\left(\mathrm{cmH}_{2} \mathrm{O}\right)\end{array}$ & $\begin{array}{c}\text { CVP } \\
\text { Median } \\
\left(\mathrm{cmH}_{2} \mathrm{O}\right)\end{array}$ & $\begin{array}{c}\text { CVP } \\
\text { Min } \\
\left(\mathrm{cmH}_{2} \mathrm{O}\right)\end{array}$ & $\begin{array}{c}\text { CVP } \\
\mathrm{Max} \\
\left(\mathrm{cmH}_{2} \mathrm{O}\right)\end{array}$ & $\begin{array}{l}\text { TAV } \\
\text { Mean } \\
(\mathrm{cm} / \mathrm{s})\end{array}$ & $\begin{array}{l}\text { TAV } \\
\text { SD } \\
(\mathrm{cm} / \mathrm{s})\end{array}$ & $\begin{array}{c}\text { TAV } \\
\text { Median } \\
(\mathrm{cm} / \mathrm{s})\end{array}$ & $\begin{array}{l}\text { TAV } \\
\text { Min } \\
(\mathrm{cm} / \mathrm{s})\end{array}$ & $\begin{array}{l}\text { TAV } \\
\text { Max } \\
\left(\mathrm{cm}^{2}\right)\end{array}$ & $\begin{array}{l}\text { CSA } \\
\text { Mean } \\
\left(\mathrm{cm}^{2}\right)\end{array}$ & $\begin{array}{l}\text { CSA } \\
\text { SD } \\
\left(\mathrm{cm}^{2}\right)\end{array}$ & $\begin{array}{c}\text { CSA } \\
\text { Median } \\
\left(\mathrm{cm}^{2}\right)\end{array}$ & $\begin{array}{l}\text { CSA } \\
\text { Min } \\
\left(\mathrm{cm}^{2}\right)\end{array}$ & $\begin{array}{l}\text { CSA } \\
\text { Max }\end{array}$ \\
\hline 1 & 10.5 & 0.68 & 10.41 & 9.15 & 11.95 & 2.09 & 4.67 & 2.64 & -6.84 & 10.29 & 0.92 & 0.03 & 0.91 & 0.86 & 0.97 \\
\hline 2 & 3.56 & 1.7 & 3.82 & -0.48 & 6.96 & 15.62 & 6.59 & 14.64 & 3.37 & 30.15 & 0.62 & 0.1 & 0.6 & 0.42 & 0.84 \\
\hline 4 & 8.52 & 1.38 & 8.59 & 5.17 & 11.49 & 10.16 & 5.16 & 11.84 & -3.47 & 19.4 & 0.89 & 0.02 & 0.88 & 0.86 & 0.95 \\
\hline 5 & 7.14 & 0.65 & 7.25 & 5.78 & 8.36 & 10.02 & 6.85 & 11.05 & -16.23 & 24.64 & 1.27 & 0.09 & 1.28 & 1.09 & 1.43 \\
\hline 6 & 7.13 & 0.97 & 7.12 & 5.3 & 9.12 & 10.41 & 3.95 & 10.68 & -2.4 & 18.78 & 1.36 & 0.09 & 1.37 & 1.13 & 1.51 \\
\hline 7 & 3.85 & 1.18 & 3.94 & 1.09 & 6.22 & 21.82 & 12.26 & 21.51 & -1.09 & 46.81 & 0.45 & 0.05 & 0.44 & 0.37 & 0.54 \\
\hline 8 & 4.46 & 1.49 & 4.7 & 0.85 & 7.51 & 8.77 & 3.91 & 8.96 & -3.75 & 15.76 & 1.36 & 0.06 & 1.36 & 1.15 & 1.47 \\
\hline 9 & 7.54 & 0.58 & 7.46 & 6.22 & 8.67 & 8.88 & 2.39 & 9.21 & 0.21 & 13.15 & 1.06 & 0.02 & 1.06 & 1.01 & 1.11 \\
\hline
\end{tabular}

Data are shown as mean, standard deviation (SD), median, minimum (min) and maximum (max). CVP, central venous pressure; TAV, time-average velocity; CSA, cross-sectional area; SD, standard deviation.

Table 2. Correlations and lag times between the CVP, TAV and IJV-CSA signals for the respective subjects.

\begin{tabular}{|c|c|c|c|c|c|c|c|c|c|}
\hline ID & $\begin{array}{l}\text { Correlation } \\
\text { between the } \\
\text { VP \& TAV signals } \\
\text { (r) }\end{array}$ & $\begin{array}{l}\text { Correlation } \\
\text { between the } \\
\text { CVP \& CSA signals } \\
\text { (r) }\end{array}$ & $\begin{array}{l}\text { Correlation } \\
\text { between the } \\
\text { CSA \& TAV signals } \\
\text { (r) }\end{array}$ & $\begin{array}{l}\text { Lag.time } \\
\text { between the } \\
\text { CVP \& TAV signals } \\
\text { (s) }\end{array}$ & $\begin{array}{l}\text { Peak lag correlation } \\
\text { between the } \\
\text { CVP \& TAV signals } \\
\text { (r)(s) }\end{array}$ & $\begin{array}{c}\text { Lag.time } \\
\text { between the } \\
\text { CVP \& CSA signals } \\
\text { (r) }\end{array}$ & $\begin{array}{l}\text { Peak lag correlation } \\
\text { between the } \\
\text { CVP \& CSA signals } \\
\text { (s) }\end{array}$ & $\begin{array}{c}\text { Lag.time } \\
\text { between the } \\
\text { CSA \& TAV signals } \\
\text { signals }\end{array}$ & $\begin{array}{l}\text { Peak lag } \\
\text { correlation between } \\
\text {; the CSA \& TAV } \\
\text { (r) }\end{array}$ \\
\hline 1 & -0.571 & 0.677 & -0.603 & -0.050 & -0.601 & -0.077 & 0.786 & 0.072 & -0.687 \\
\hline 2 & 0.115 & 0.136 & -0.684 & -0.211 & -0.726 & -0.179 & 0.648 & -0.014 & -0.690 \\
\hline 4 & -0.112 & -0.112 & -0.722 & -0.210 & $-0.447 \#$ & -0.159 & $0.263 \#$ & -0.042 & -0.777 \\
\hline 5 & 0.481 & -0.355 & -0.407 & -0.118 & -0.603 & -0.112 & 0.808 & 0.012 & $-0.410 \#$ \\
\hline 6 & 0.342 & 0.148 & 0.342 & -0.156 & $-0.021 \#$ & -0.031 & 0.352 & 0.078 & -0.329 \\
\hline 7 & 0.219 & -0.743 & -0.453 & -0.034 & -0.813 & -0.150 & 0.713 & 0.130 & -0.804 \\
\hline 8 & $-0.059 *$ & 0.180 & 0.166 & -0.148 & $-0.163 \#$ & -0.127 & $0.109 \#$ & -0.199 & $-0.077^{\star}$ \\
\hline 9 & -0.175 & -0.329 & -0.167 & -0.102 & -0.740 & -0.270 & 0.292 & 0.098 & -0.462 \\
\hline Mean & 0.030 & -0.050 & -0.316 & -0.129 & -0.514 & -0.138 & 0.496 & 0.017 & -0.530 \\
\hline SD & 0.333 & 0.433 & 0.396 & 0.066 & 0.2860 .071 & 0.272 & 0.105 & 0.254 & \\
\hline
\end{tabular}

All r-values significant at $\mathrm{P}=0.001$, except for * which was not significant; thigher correlation value in the opposite direction observed but not recorded in table. Data are shown as mean, standard deviation (SD), median, minimum (min) and maximum (max). CVP, central venous pressure; TAV, time-average velocity; CSA, cross-sectional area; SD, standard deviation.

Table 3. Correlations between the central venous pressure, time-average velocity and internal jugular vein cross-sectional area (IJV-CSA) autocorrelation signals for the respective subjects, together with the periodicity of the IJV-CSA autocorrelation signal.

\begin{tabular}{|c|c|c|c|c|}
\hline ID & $\begin{array}{l}\text { Correlation between } \\
\text { the CVP \& TAV } \\
\text { autocorrelation signals } \\
\text { (r) }\end{array}$ & $\begin{array}{l}\text { Correlation between } \\
\text { the CVP \& CSA } \\
\text { autocorrelation signals } \\
\text { (r) }\end{array}$ & $\begin{array}{l}\text { Correlation between } \\
\text { the CSA \& TAV } \\
\text { autocorrelation signals } \\
\text { (r) }\end{array}$ & $\begin{array}{c}\text { Periodicity } \\
\text { of CSA } \\
\text { autocorrelation signal } \\
(\mathrm{Hz})\end{array}$ \\
\hline 1 & 0.925 & 0.926 & 0.944 & 1.066 \\
\hline 2 & 0.906 & 0.916 & 0.833 & 1.049 \\
\hline 4 & 0.770 & 0.584 & 0.792 & 1.370 \\
\hline 5 & 0.854 & 0.957 & 0.770 & 1.021 \\
\hline 6 & 0.498 & 0.726 & 0.440 & 1.028 \\
\hline 7 & 0.872 & 0.803 & 0.931 & 1.040 \\
\hline 8 & 0.357 & 0.583 & 0.532 & 1.054 \\
\hline 9 & 0.927 & 0.373 & 0.588 & 0.869 \\
\hline Mean & 0.764 & 0.734 & 0.729 & 1.062 \\
\hline SD & 0.217 & 0.207 & 0.187 & 0.139 \\
\hline
\end{tabular}

CVP, central venous pressure; TAV, time-average velocity; CSA, cross-sectional area; SD, standard deviation. 
and CSA: mean (SD) r=0.734 (0.207); and CSA and TAV: mean (SD) r=0.729 (0.187)], with the mean periodicity frequency of the CSA signal being $1.062 \mathrm{~Hz}(\mathrm{SD}=0.139 \mathrm{~Hz})$.

Examples of the autocorrelation signals are shown in Figures 1B and 2B, which show the autocorrelation plots for the respective signals for subjects 2 (typical) and 8 (atypical). From these it can be seen that for the typical subject (Subject 2, Figure 1B) the IJV-CSA autocorrelation signal was smooth with a regular periodicity of $1.049 \mathrm{~Hz}$, whereas with the atypical subject (Subject 8 , Figure 2B) the CSA signal was more irregular, but with a similar periodicity (i.e., $1.054 \mathrm{~Hz}$ ). For Subject 2 the CVP and TAV autocorrelation signals were highly positively correlated $(\mathrm{r}=0.906, \mathrm{P}<0.001)$, and although having a more complex profile than the CSA autocorrelation signal, still exhibited a similar overall periodicity to the CSA signal. By contrast, for Subject 8 the CVP and TAV autocorrelation signals were much more weakly correlated $(\mathrm{r}=0.357$, $\mathrm{P}<0.001)$ and exhibited a different periodicity to the CSA signal. The results of the spectral analysis are presented in Table 4 and illustrated in Figures $1 \mathrm{C}$ and 2C.

From Table 4 it can be seen that for many of the subjects the frequency spectrums of the CVP, TAV and CSA signals were dominated by the same frequencies. For example, in four of the subjects (subjects 1,2,5 and 7) the dominant frequency (i.e. Freq. 1) was the same for CVP, TAV and CSA signals. In Subject 4 the $0.999 \mathrm{~Hz}$ frequency was dominant (Freq. 1) for the TAV and CSA signals, while for the CVP signal it was second dominant (Freq. 2). Similarly, in Subject 9 the CVP and TAV signals are dominated by the same frequencies. Only in subjects 6 and 8 were the spectral results atypical. Comparison between the periodograms in Figures $1 \mathrm{C}$ and $2 \mathrm{C}$ illustrates this phenomenon very well. For the typical subject (Subject 2) the periodogram in Figure 1C reveals that all three signals are influenced by strong peaks at $0.999,1.979$ and $2.969 \mathrm{~Hz}$, whereas for Subject 8 the spectral peaks of the three signals are not aligned.

\section{Discussion}

From the analysis above it can be seen that while it is difficult to interpret the CVP, TAV and CSA signals in the time domain, a much clearer picture emerges when autocorrelation and Fourier analysis are performed. In the frequency domain, we found that with the exception of Subjects 6 and 8 , the periodograms for the respective signals in the other subjects were dominated by discrete frequencies at approximately 1 and 2 $\mathrm{Hz}$, with additional higher frequencies also making a major contribution in some subjects, as illustrated by Subject 2 (Figure 1C). Furthermore, we found that the higher frequencies (i.e., $>1 \mathrm{~Hz}$ ) tended to be attenuated more in the CSA signal compared with the other two signals, as illustrated in Figure 1C. As such, this explains why the CSA autocorrelation signals tended to be characterized by a smooth undulating curve with a regular periodicity of about $1 \mathrm{~Hz}$ (e.g., Figure 1B), reflecting the underlying fundamental cardiac frequency of the heart. By comparison, the autocorrelation curves for the CVP and TAV were influenced to a greater extent by the higher frequencies, resulting in more complex curves, but still with a general periodicity of about $1 \mathrm{~Hz}$, as illustrated in Figure 1B. This suggests that the mechanical work required to move the IJV vessel walls attenuates the higher frequencies, with the result that the IJV pulse primarily reflects the fundamental cardiac frequency. Notwithstanding this, there were clearly cases where the above explanation did not apply. In Subjects 6 and 8 the spectra of the three signals did not align, as can be seen in Figure 2C, with the result that for these subjects the correlations between the respective autocorrelation signals were
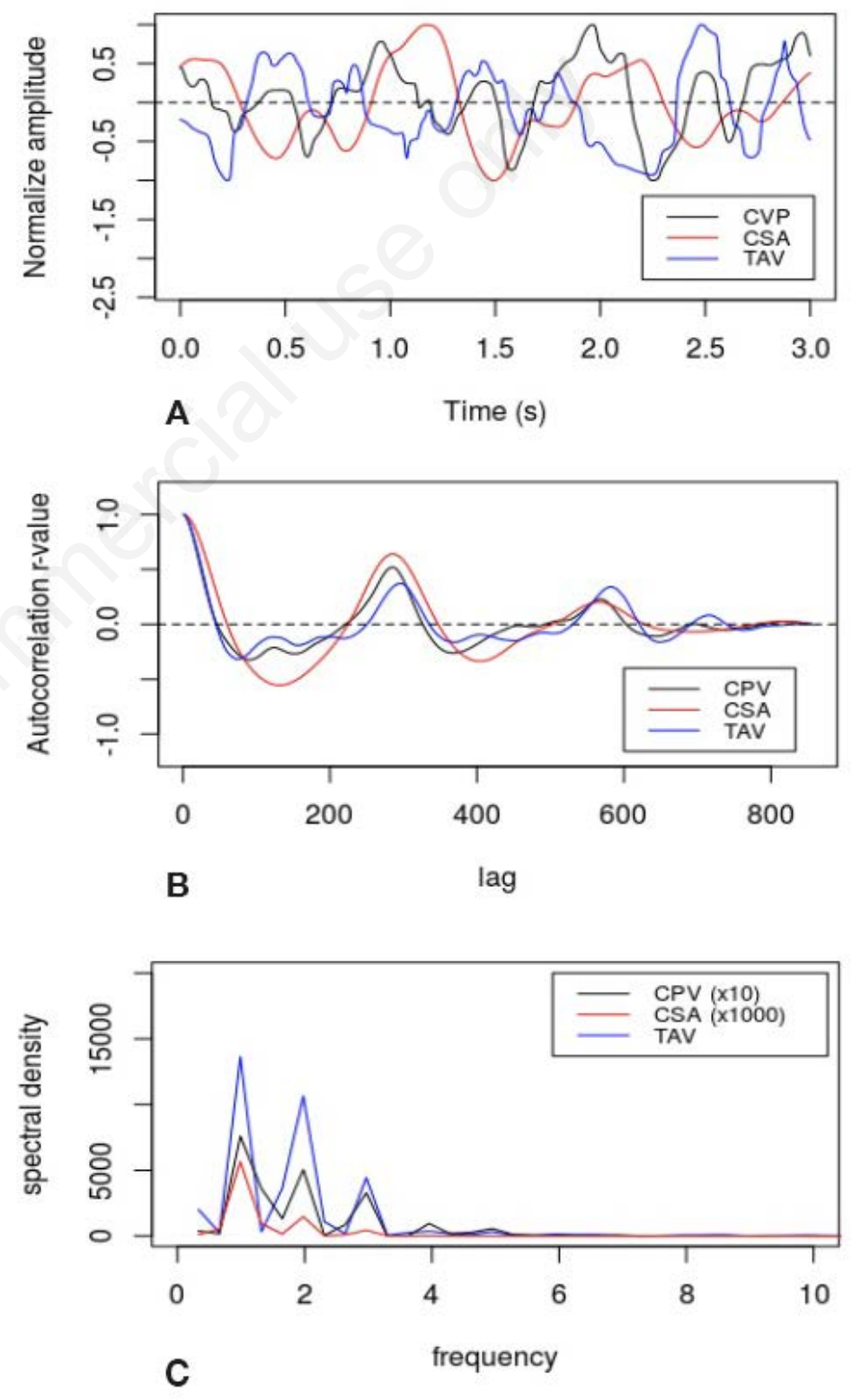

Figure 1. A) Normalized internal jugular vein cross-sectional area (CSA), time-average velocity (TAV) and central venous pressure (CVP) time-series signals; B) autocorrelation signals; and C) periodogram of the frequency spectra for Subject 2 (i.e., typical subject). 
much weaker (e.g., Figure 2B). Although the reasons are unknown, it may be argued that the observed misalignment in the frequency domain among the various signals is an indicator of some underlying pathophysiological condition. Interestingly, Subjects 6 and 8 were the only two in our sample being affected by heart disease. For most of the subjects the periodograms (not shown) exhibited a strong peak in the CVP and TAV spectral signals at approximately 2 $\mathrm{Hz}$, with many having discrete peaks at frequencies $>2 \mathrm{~Hz}$, as illustrated in Figure 1C. These higher frequency components probably represent the harmonics of the fundamental resting cardiac frequency (i.e., approximately $1 \mathrm{~Hz}$ ). Pressure waves in the cardiovascular system can be characterized as complex periodic sine waves, which are a summation of a series of simple sine waves of differing amplitudes and frequencies, which represent the natural harmonics of a fundamental frequency. ${ }^{23}$ Although not dominant, it is noticeable that many subjects had a significant component frequency at around $0.33 \mathrm{~Hz}$, which is consistent with a respiratory rate of approximately 20 breaths per minute. CVP is known to be affected by intrathoracic pressure..$^{21,24,25}$ During inspiration, the intrathoracic pressure becomes more negative and the CVP drops, increasing venous blood return from the body to the right atrium via the superior and inferior venae cavae. ${ }^{25}$ While the data was more difficult to interpret in the time domain, there appeared to be a moderately strong inverse correlation [mean (SD) $\mathrm{r}=-$ $0.506(0.207)]$ between the CSA and TAV signals in the six typical subjects, whereas this relationship was weakly positive [mean (SD) $\mathrm{r}=0.254(0.124)$ ] in subjects 6 and 8 . This finding suggests that in the typical subjects the behavior of the IJVs broadly conformed to Bernoulli's theorem, ${ }^{26}$ which states that a decrease in static pressure occurs simultaneously with an increase in the speed of a fluid flowing through the vessel. Given that the thin-walled IJVs readily respond to changes in static pressure, it is not surprising that an inverse correlation was observed between CSA and TAV. Therefore, as the blood velocity in the IJVs reduced, so the static pressure increased, with the result that the vessel CSA increased. Conversely, as TAV increased, so the static pressure fell and the CSA reduced. Nevertheless, we were surprised to find in the atypical subjects (subjects 6 and 8) that this relationship was positive. Though this finding is difficult to explain, it is noticeable that in these two subjects the mean IJVCSA was larger than in the other six subjects. Although the instantaneous correlation results for the between CVP and TAV, and CVP and CSA were inconclusive, when cross-correlation analysis was performed on these signals (Table 2), a clear negative lag correlation [mean (SD) $\mathrm{r}=-0.514(0.286)]$ was observed between the CVP and TAV signals, and a positive lag correlation [mean (SD) $r=0.496 \quad(0.272)]$ was observed between the CVP and CSA signals. Both TAV and CSA signals lagged behind the CVP signal by approximately 0.13-0.14 s, although there was no significant difference between the observed lag times. Collectively, this confirms that the CVP 'drives' much of the behavior of IJV pulse, with the oscillations in IJV-CSA corresponding the changes in venous pressure that occur due to the contraction and relaxation of the right atrium and ventricle, and the opening and closing of the tricuspid value. ${ }^{27,28}$ When the CVP rises, it causes, after a short delay, the blood velocity in the IJVs to decrease, presumably because the pressure gradient driving the fluid flow towards the heart decreases in magnitude. Likewise, as the CVP rises, so it reduces the blood velocity and increases the static pres-
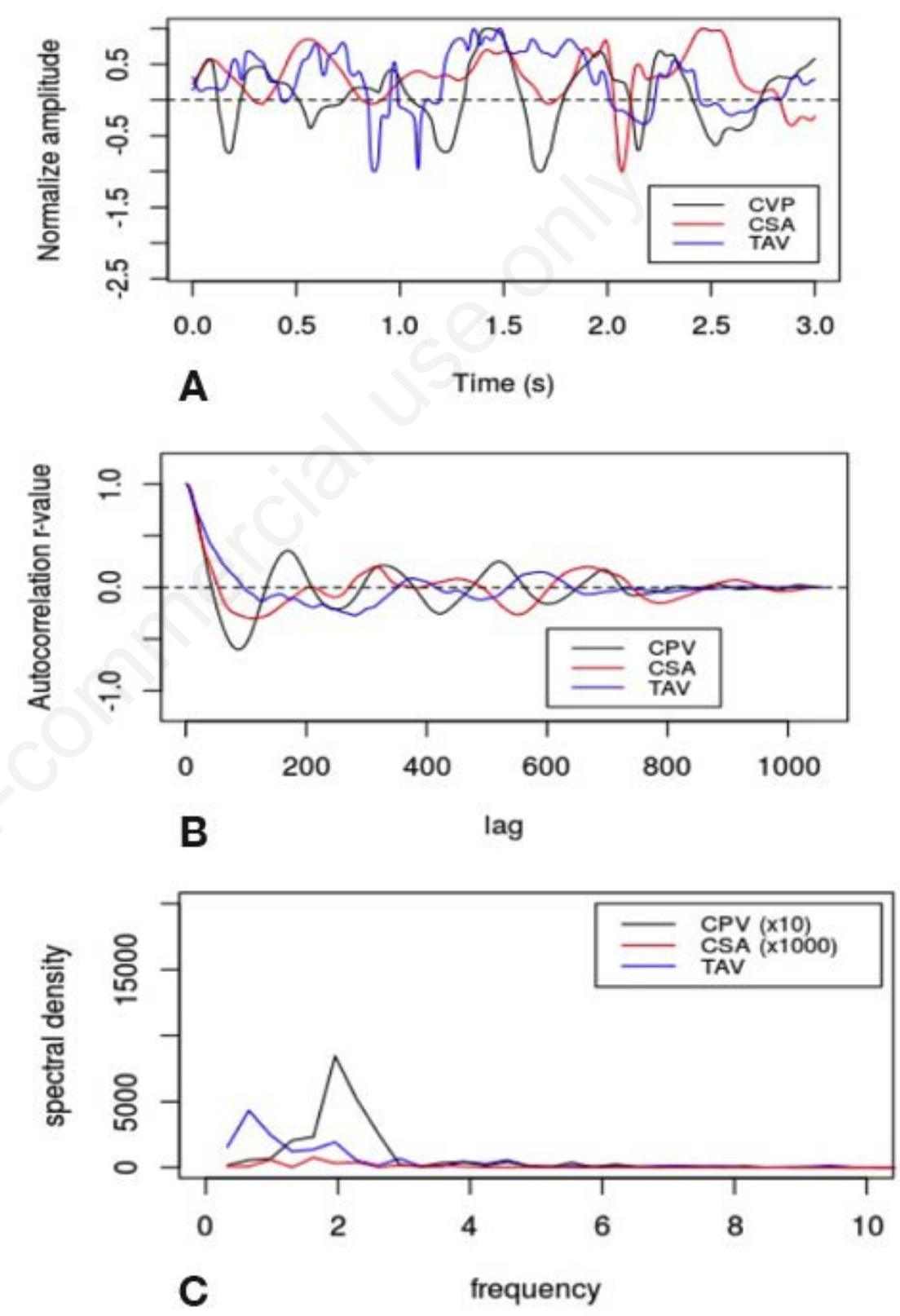

Figure 2. A) Normalized internal jugular vein cross-sectional area (CSA), time-average velocity (TAV) and central venous pressure (CVP) time-series signals; $B$ ) autocorrelation signals; and C) periodogram of the frequency spectra for Subject 8 (i.e. atypical subject). 
sure in the IJVs, with the result that the CSA of the vessels also increases.

The flow of blood entering the IJVs from the cranium is known to be regulated by the intracranial windkessel mechanism. ${ }^{29,30}$ This mechanism coordinates the volumetric changes in CSF and arterial and venous blood that occur in the cranium over the $\mathrm{CC}^{7}$, ensuring the smooth flow of blood through the brain parenchyma. ${ }^{29}$ These volumetric changes are ultimately driven by the arterial flow from the left ventricle of the heart. As such, blood flow in the IJVs is not only influenced by 'pull' of the right atrium, but also be the 'push' of the left ventricle, in a highly coordinated physiological process, which can be affected by many factors. With respect to this, any rise in CVP will tend to increase the CSA of the IJVs. Although the dynamics of the brain-heart connection via the IJVs is not fully understood, it is likely that alterations (anomalies) in the IJV pulse will reflect not only changes in the right side of the heart, but also changes in the intracranial fluid dynamics. While, it has been shown that constriction of the IJVs is associated with increased CSF pulsatility in the $\operatorname{AoS}^{6,31,32}$ virtually no work has been undertaken to assess the impact of neurological disease on the JVP itself. Therefore, there is pressing need to better understand the impact that neurological pathology has on the dynamics of the cerebral venous drainage system, as the peripheral venous vessel are accessible and can be easily monitored non-invasively with Doppler ultrasound. Albeit that a major limitation of our study is the small number of subjects sampled, it is noticeable that the study cohort broadly divided into six typical and two atypical sub-groups. This in itself is an interesting finding because it highlights the considerable variability that exists with respect to the IJVs signals. Therefore, further work will be required to better understand: i) what is typical; ii) why certain individuals exhibit atypical characteristics; and iii) the clinical relevance of atypical IJVs. Furthermore, although the present study was only concerned with the relationship between the IJVs and the heart, it is anticipated that the knowledge acquired from this work will inform future studies investigating pathologies of both the heart and the brain. It is therefore recommended that future work be undertaken to evaluate the impact that neurological pathologies might have on the IJP, as this would increase the usefulness of the IJP as a clinical diagnostic indicator.

Table 4. Top five spectral frequencies, together with corresponding amplitudes, for the: central venous pressure, time-average velocity and internal jugular vein cross-sectional area signals from the respective subjects.

\begin{tabular}{|c|c|c|c|c|c|c|c|c|c|c|}
\hline \multirow[b]{2}{*}{ ID } & \multirow[b]{2}{*}{ Freq.1 } & \multirow[b]{2}{*}{ Freq.2 } & \multirow[b]{2}{*}{ Freq.3 } & \multicolumn{3}{|c|}{ Central venous pressure } & \multirow[b]{2}{*}{ Amp.2 } & \multirow[b]{2}{*}{ Amp.3 } & \multirow[b]{2}{*}{ Amp.4 } & \multirow[b]{2}{*}{ Amp.5 } \\
\hline & & & & Freq.4 & Freq.5 & Amp.1 & & & & \\
\hline 1 & 0.987 & 2.960 & 1.316 & 0.658 & 3.947 & 124.152 & 22.582 & 21.491 & 20.199 & 16.161 \\
\hline 2 & 0.990 & 1.979 & 1.319 & 2.969 & 1.649 & 759.538 & 504.922 & 360.520 & 327.999 & 131.321 \\
\hline 4 & 1.998 & 0.999 & 2.331 & 4.995 & 6.327 & 824.219 & 316.540 & 94.664 & 92.471 & 74.685 \\
\hline 5 & 0.994 & 1.988 & 2.981 & 0.745 & 0.497 & 182.178 & 24.561 & 18.237 & 13.210 & 8.839 \\
\hline 6 & 0.653 & 0.980 & 1.960 & 1.633 & 1.307 & 289.366 & 137.075 & 80.719 & 69.909 & 53.546 \\
\hline 7 & 0.994 & 1.987 & 4.968 & 2.981 & 0.331 & 286.873 & 257.847 & 36.859 & 34.223 & 32.540 \\
\hline 8 & 1.956 & 2.281 & 2.607 & 1.630 & 1.304 & 844.797 & 519.071 & 258.122 & 232.484 & 205.475 \\
\hline 9 & 1.952 & 0.976 & 2.603 & 0.325 & 0.651 & 142.200 & 32.155 & 16.893 & 9.521 & 8.156 \\
\hline ID & Freq.1 & \multicolumn{8}{|c|}{ Time-average velocity } & Amp.5 \\
\hline 1 & 0.987 & 1.316 & 2.302 & 3.289 & 2.960 & 8897.6 & 2123.6 & 599.7 & 560.7 & 547.1 \\
\hline 2 & 0.990 & 1.979 & 2.969 & 1.649 & 0.330 & 13638.9 & 10644.3 & 4434.5 & 3642.0 & 2005.7 \\
\hline 4 & 0.999 & 1.998 & 1.332 & 1.665 & 2.997 & 7898.6 & 7396.8 & 3120.3 & 2788.4 & 2116.2 \\
\hline 5 & 0.994 & 1.988 & 0.745 & 0.497 & 1.739 & 8856.6 & 3883.2 & 2623.7 & 2478.5 & 2287.8 \\
\hline 6 & 1.633 & 0.327 & 0.653 & 1.307 & 0.980 & 2669.6 & 1773.9 & 1655.1 & 1604.0 & 1080.2 \\
\hline 7 & 0.994 & 1.987 & 0.662 & 0.331 & 1.325 & 55897.7 & 19915.2 & 11121.9 & 4055.7 & 1370.5 \\
\hline 8 & 0.652 & 0.978 & 1.956 & 0.326 & 1.630 & 4326.7 & 2468.4 & 1922.7 & 1564.5 & 1362.8 \\
\hline 9 & 1.952 & 0.976 & 2.277 & 1.301 & 4.880 & 1864.0 & 816.1 & 457.6 & 416.7 & 182.5 \\
\hline \multicolumn{10}{|c|}{ Internal jugular vein cross-sectional area } & Amp.5 \\
\hline 1 & 0.987 & 1.644 & 0.658 & 0.329 & 2.960 & 0.451 & 0.056 & 0.050 & 0.036 & 0.031 \\
\hline 2 & 0.990 & 1.979 & 1.319 & 0.660 & 2.969 & 5.640 & 1.461 & 0.942 & 0.525 & 0.443 \\
\hline 4 & 0.999 & 0.666 & 1.998 & 1.332 & 1.665 & 0.321 & 0.084 & 0.067 & 0.030 & 0.013 \\
\hline 5 & 0.994 & 1.988 & 1.491 & 0.497 & 2.981 & 3.398 & 0.456 & 0.203 & 0.166 & 0.156 \\
\hline 6 & 0.980 & 0.327 & 1.307 & 1.633 & 0.653 & 3.959 & 0.823 & 0.595 & 0.514 & 0.390 \\
\hline 7 & 0.994 & 1.987 & 0.331 & 1.325 & 0.662 & 1.084 & 0.179 & 0.060 & 0.044 & 0.039 \\
\hline 8 & 1.630 & 0.978 & 2.281 & 1.956 & 2.933 & 0.763 & 0.599 & 0.418 & 0.328 & 0.177 \\
\hline 9 & 1.301 & 0.976 & 2.928 & 2.603 & 1.952 & 0.075 & 0.060 & 0.037 & 0.032 & 0.027 \\
\hline
\end{tabular}

Freq., frequency; Amp., amplitude. 


\section{Conclusions}

Compared with working purely in the time-domain, it is much easier to identify atypical anomalies in the JVP using spectral and autocorrelation analysis. In typical subjects, although the higher harmonic frequencies are greatly attenuated in the IJVCSA signals, the autocorrelograms and periodograms for the CVP, TAV and CSA signals are aligned and are dominated by the same underlying frequencies. By comparison, in atypical subjects the respective autocorrelogram and periodogram plots are not aligned. The reasons for this atypical behavior are not known and further work will be required to better understand the clinical relevance of this finding. Finally, the influence of changes in posture out of the breathing needs to be better compared in order to define the better protocol for signal acquisition. ${ }^{33,34}$

\section{References}

1. Garg N, Garg N. Jugular venous pulse: an appraisal. J Indian Acad Clin Med 2000;1;261-9.

2. Chua Chiaco JM, Parikh NI, Fergusson DJ. The jugular venous pressure revisited. Cleve Clin J Med 2013;80:638-44.

3. Applefeld MM. The jugular venous pressure and pulse contour. In: Walker HK, Hall WD, Hurst, JW, eds. Clinical methods: the history, physical, and laboratory examinations. Boston, MA: Butterworth; 1990. pp 107-111.

4. Drazner MH, Rame JE, Stevenson LW, Dries DL. Prognostic importance of elevated jugular venous pressure and a third heart sound in patients with heart failure. N Engl J Med 2001;345:574-81.

5. Beggs CB, Magnano C, Shepherd SJ, et al. Aqueductal cerebrospinal fluid pulsatility in healthy individuals is affected by impaired cerebral venous outflow. J Magn Reson Imaging 2014;40:1215-22.

6. Hatt A, Cheng S, Tan K, et al. MR elastography can be used to measure brain stiffness changes as a result of altered cranial venous drainage during jugular compression. AJNR Am J Neuroradiol 2015;36:1971-7.

7. Lagana MM, Shepherd SJ, Cecconi P, Beggs CB. Intracranial volumetric changes govern cerebrospinal fluid flow in the Aqueduct of Sylvius in healthy adults. Biomed Signal Process Control 2017;36:84-92.

8. Ward KR, Tiba MH, Barbee RW, et al. A new noninvasive method to deter- mine central venous pressure. Resuscitation 2006;70:238-46.

9. Brennan JM, Blair JE, Goonewardena $\mathrm{S}$, et al. A comparison by medicine residents of physical examination versus hand-carried ultrasound for estimation of right atrial pressure. Am J Cardiol 2007;99:1614-6.

10. Sanfilippo F, Noto A, Martucci G, et al. Central venous pressure monitoring via peripherally or centrally inserted central catheters: a systematic review and meta-analysis. J Vasc Access 2017; 18:273-8.

11. McGee DC, Gould MK. Preventing complications of central venous catheterization. $\mathrm{N}$ Engl $\mathrm{J}$ Med 2003;348:1123-33.

12. Sisini F, Tessari M, Gadda G, et al. An ultrasonographic technique to assess the jugular venous pulse: a proof of concept. Ultrasound Med Biol 2015;41:1334-41.

13. Sisini F, Tessari M, Menegatti E, et al. Clinical applicability of assessment of jugular flow over the individual cardiac cycle compared with current ultrasound methodology. Ultrasound Med Biol 2016;42:1750-63.

14. Hossein-Nejad H, Mohammadinejad P, Ahmadi F. Internal jugular vein/common carotid artery cross-sectional area ratio and central venous pressure. J Clin Ultrasound 2016;44:312-8.

15. Ogum C, Hariharan S, Chen D. Noninvasive central venous pressure estimation by ultrasound guided internal jugular vein cross-sectional area measurement. Biomed Phys Eng Express 2016;2:025004.

16. Bano S, Qadeer A, Akhtar A, et al. Measurement of internal jugular vein and common carotid artery diameter ratio by ultrasound to estimate central venous pressure. Cureus 2018;10:e2277.

17. Zamboni P, Sisini F, Menegatti E, et al. Ultrasound monitoring of jugular venous pulse during space missions: proof of concept. Ultrasound Med Biol 2018;44:726-33.

18. Zamboni P, Malagoni AM, Menegatti E, et al. Central venous pressure estimation from ultrasound assessment of the jugular venous pulse. PLoS One 2020; 15:e0240057.

19. Smith RN, Nolan JP. Central venous catheters. BMJ 2013;347:f6570.

20. Tavoni V. Technical note for post processing of jugular venous pulse, central venous pressure and velocity trace. Veins and Lymphatics 2020;9:8268.

21. Zamboni P, Menegatti E, Pomidori L, et al. Does thoracic pump influence the cerebral venous return? J Appl Physiol (1985) 2012;112:904-10.

22. Chatfield $\mathrm{C}$. The analysis of time series: an introduction. 4th ed. Boca Raton, FL: Chapman and Hall; 1989.

23. Welch JP, D'Ambra MN. Hemodynamic monitoring, In: Kofke WA, Levy JH, eds. Postoperative critical care procedures of the Massachusetts General Hospital. Volume 146. Boston, MA: Little, Brown and Company; 1986. pp 146.

24. Triedman JK, Saul JP. Blood pressure modulation by central venous pressure and respiration. Buffering effects of the heart rate reflexes. Circulation 1994;89:169-79.

25. Gelman S. Venous function and central venous pressure: a physiologic story. Anesthesiology. 2008;108:735-48.

26. Batchelor CK, Batchelor GK. An introduction to fluid dynamics. Cambridge: Cambridge University Press; 1967.

27. Lee CH, Xiao HB, Gibson DG. Jugular venous 'a' wave in dilated cardiomyopathy: sign of abbreviated right ventricular filling time. Br Heart J 1991;65:3425.

28. Fukuda N, Oki T, Iuchi A, et al. Right heart flow dynamics after tricuspid valve annuloplasty. Characteristics and time course. Jpn Heart J 1998;39:33946.

29. Bateman GA, Levi CR, Schofield P, et al. The venous manifestations of pulse wave encephalopathy: windkessel dysfunction in normal aging and senile dementia. Neuroradiology 2008;50:491-7.

30. Zou R, Park EH, Kelly EM, et al. Intracranial pressure waves: characterization of a pulsation absorber with notch filter properties using systems analysis: laboratory investigation. J Neurosurg Pediatr 2008;2:83-94.

31. Zamboni P, Menegatti E, WeinstockGuttman B, et al. The severity of chronic cerebrospinal venous insufficiency in patients with multiple sclerosis is related to altered cerebrospinal fluid dynamics. Funct Neurol 2009;24:133-38.

32. Magnano C, Schirda C, WeinstockGuttman B, et al. Cine cerebrospinal fluid imaging in multiple sclerosis. J Magn Reson Imaging 2012;36:825-34.

33. Zamboni P, Tavoni V, Sisini F, et al. Venous compliance and clinical implications. Veins and Lymphatics 2018;7:7367.

34. Beggs CB. Cerebral venous outflow and cerebrospinal fluid dynamics. Veins and Lymphatics 2014;3:1867. 